\title{
Neck Flexion Induces Larger Deformation of the Brain Than Extension at a Rotational Acceleration, Closed Head Trauma
}

\author{
Hans-Arne Hansson, ${ }^{1}$ Ulrika Krave, ${ }^{2}$ Svante Höjer, ${ }^{3}$ and Johan Davidsson ${ }^{2}$ \\ ${ }^{1}$ Institute of Biomedicine, Sahlgren Academy, University of Gothenburg, P.O. Box 440, 40530 Gothenburg, Sweden \\ ${ }^{2}$ Department of Applied Mechanics, Chalmers University of Technology, 41296 Gothenburg, Sweden \\ ${ }^{3}$ GU Holding, University of Gothenburg, 40530 Gothenburg, Sweden
}

Correspondence should be addressed to Hans-Arne Hansson; hans-arne.hansson@gu.se

Received 16 June 2014; Revised 16 September 2014; Accepted 7 October 2014; Published 3 November 2014

Academic Editor: John B. Redell

Copyright (C) 2014 Hans-Arne Hansson et al. This is an open access article distributed under the Creative Commons Attribution License, which permits unrestricted use, distribution, and reproduction in any medium, provided the original work is properly cited.

A closed head trauma induces incompletely characterized temporary movement and deformation of the brain, contributing to the primary traumatic brain injury. We used the pressure patterns recorded with light-operated miniature sensors in anaesthetized adult rabbits exposed to a sagittal plane rotational acceleration of the head, lasting $1 \mathrm{~ms}$, as a measure of brain deformation. Two exposure levels were used and scaled to correspond to force levels reported to cause mild and moderate diffuse injury in an adult man, respectively. Flexion induced transient, strong, extended, and predominantly negative pressures while extension generated a short positive pressure peak followed by a minor negative peak. Low level flexion caused as strong, extended negative pressures as did high level extension. Time differences were demonstrated between the deformation of the cerebrum, brainstem, and cerebellum. Available X-ray and MRI techniques do not have as high time resolution as pressure recordings in demonstrating complex, sequential compression and stretching of the brain during a trauma. The exposure to flexion caused more protracted and extensive deformation of the brain than extension, in agreement with a published histopathological report. The severity and extent of the brain deformation generated at a head trauma thus related to the direction at equal force.

\section{Introduction}

A closed head trauma may result in traumatic brain injury (TBI), and its consequences constitute a large burden for the victims, their families, and the society [1-5]. The relation between the external loading of the head and the response in the brain, resulting in damage, during an impact lasting just milliseconds needs further clarification. Inertial shearing deformation of the brain is considered to be a primary cause of injury [6-14] and generates temporary pressures in the brain parenchyma, as demonstrated in, for example, postmortem human subjects and in nonhuman primates [15-17]. Anderson et al. [18] reported correlations between applied force, dynamic pressures, and histopathological changes at a lateral head impact. The forces applied at a closed head impulse have been proposed to possibly induce cavitation at interfaces [19-23].
The aim of the present study was to elucidate the importance of the direction of a sagittal plane rotational acceleration trauma to the head and neck for the deformation of the brain, which thereby induces brain concussion, also named mild traumatic brain injury (TBI) [10, 12-14, 17]. We consider that the direction of the force at a head trauma is likely to influence the resulting primary brain damage, which is generated during as short time as just a few milliseconds [6, 7, 24-27]. Pudenz and Shelden [28] were the first to demonstrate that at a trauma the brain moves within the skull and is deformed, as subsequently repeatedly confirmed $[6,7$, $10,11,29,30]$. A number of approaches have been reported to further characterize the movement and deformation of the brain at the exposure to a trauma by, for example, high speed photography of the brain through a window in the calvarium, the use of flash X-ray of implanted lead particles, or advanced biplanar X-ray system combined with embedded 
TABLE 1: Number of animals, exposures and results.

\begin{tabular}{|c|c|c|c|c|c|c|c|c|}
\hline \multirow{2}{*}{$\begin{array}{l}\text { Impact condition, number } \\
\text { of animals }{ }^{1}\end{array}$} & \multicolumn{3}{|c|}{ Acceleration $\left(\mathrm{krad} / \mathrm{s}^{2}\right)$} & \multirow{2}{*}{ Location of transducer } & \multirow{2}{*}{ Animals, $n$} & \multirow{2}{*}{ Tests, $n$} & \multicolumn{2}{|c|}{ Peak Pressure (bar) } \\
\hline & Mean & Std. dev. ${ }^{4}$ & $n^{2}$ & & & & Mean $^{4}$ & Std. dev. ${ }^{4}$ \\
\hline \multirow{2}{*}{$\begin{array}{l}\text { High Flexion }{ }^{3} \\
n=4\end{array}$} & \multirow{2}{*}{208,0} & \multirow{2}{*}{ n.a. } & \multirow{2}{*}{1} & Brainstem & 1 & 1 & $-0,47$ & n.a. \\
\hline & & & & Basal ganglia & 1 & 1 & n.a & n.a. \\
\hline \multirow{5}{*}{$\begin{array}{l}\text { Low Flexion } \\
n=8\end{array}$} & \multirow{5}{*}{96,3} & \multirow{5}{*}{15,0} & \multirow{5}{*}{52} & Brainstem & 7 & 48 & $-0,38$ & 0,20 \\
\hline & & & & Basal ganglia & 6 & 30 & $-0,49$ & 0,16 \\
\hline & & & & Paraflocculus & 1 & 3 & $-0,20$ & 0,05 \\
\hline & & & & Cerebellum & 1 & 1 & $-0,15$ & n.a \\
\hline & & & & Angled in Basal ganglia & 1 & 12 & - & - \\
\hline \multirow{4}{*}{$\begin{array}{l}\text { High Extension } \\
n=10\end{array}$} & \multirow{4}{*}{$-184,5$} & \multirow{4}{*}{14,5} & \multirow{4}{*}{20} & Brainstem & 3 & 6 & 0,29 & 0,10 \\
\hline & & & & Basal ganglia & 4 & 8 & 0,34 & 0,18 \\
\hline & & & & Hippocampus & 6 & 13 & 0,62 & 0,24 \\
\hline & & & & Parieto-temporal cortex & 5 & 12 & 0,48 & 0,34 \\
\hline \multirow{4}{*}{$\begin{array}{l}\text { Low Extension } \\
n=12\end{array}$} & \multirow{4}{*}{$-95,0$} & \multirow{4}{*}{6,6} & \multirow{4}{*}{37} & Brainstem & 4 & 8 & 0,51 & 0,15 \\
\hline & & & & Basal ganglia & 4 & 18 & 0,30 & 0,16 \\
\hline & & & & Hippocampus & 8 & 24 & 0,25 & 0,14 \\
\hline & & & & Parieto-temporal cortex & 8 & 23 & 0,33 & 0,19 \\
\hline
\end{tabular}

${ }^{1}$ The total number of animals exposed to sagittal rotational acceleration impulses was 24 . Three animals were exposed to a single high level flexion tests, one animal was exposed to multiple low and high level flexion tests and four animals were exposed to multiple low level flexion tests. Three animals were exposed to multiple low level extension tests, six animals were exposed to multiple low and high level extension tests, three animals were exposed to a single high level extension test and one animal was exposed to multiple high level extension tests. Finally, three animals were exposed to multiple low level flexion and extension tests.

${ }^{2}$ The total number of experiments that provided useful data was 110 .

${ }^{3}$ The three animals that were exposed to a single high level flexion tests did not provide peak pressure data. All transducers installed bottomed out; the underpressure was as least in the range of -0.45 bar. As such these test confirm the data provided by the single test that provided brainstem pressure data.

${ }^{4}$ n.a.: not available.

neutral-density particles as well as with the aid of MRI $[6,7,11,13,28-32]$. Finite element analyses indicate that head impacts induce complex displacement and deformation resulting in compression and strain in the brain $[12,23$, 33]. We have used a different approach to elucidate what happens in the brain parenchyma during the very short time, usually in the order of milliseconds, when a sagittal plane closed head rotational acceleration impulse forces the brain to transiently move and be deformed, thereby contributing to the primary brain injury $[34,35]$. High speed recordings of the pressures generated by the mechanical events taking place in the brain parenchyma at a head and neck trauma were used by utilizing sensitive and flexible miniature pressure sensors, operated by light, and enabling high sampling rate and resolution. At neck flexion, the brain and spinal cord will initially be stretched and then after a short lag, perhaps including oscillations, regain the position in the skull and spine. In contrast, at extension of the neck, the frontal and temporal brain lobes especially will be compressed, and the brainstem, the cerebellum, and the spinal cord are as well affected. Another goal was to investigate whether the inertial displacement and deformation of the brain and spinal cord in an intact cranium and vertebral column, lasting just milliseconds, generated complex pressure patterns differing for flexion versus extension. Further, we aimed to elucidate if differences could be demonstrated between the cerebrum, brainstem, and cerebellum as judged from the recorded pressure patterns.

\section{Materials and Methods}

2.1. Ethical Considerations. The experiments were approved by the Regional Animal Experiments Ethics Committee and performed in accordance with Swedish and European Union guidelines (Directive 2010/63/EU). Care was taken to minimize the number of animals and their suffering.

2.2. Test Procedure. Two sagittal rotational acceleration rates were used at flexion, $96.3 \pm 15.0 \mathrm{krad} \cdot \mathrm{s}^{-2}$ and $208 \mathrm{krad} \cdot \mathrm{s}^{-2}$, and at extension, $-95.0 \pm 6.6 \mathrm{krad} \cdot \mathrm{s}^{-2}$ and $-184.5 \pm 14.5 \mathrm{krad} \cdot \mathrm{s}^{-2}$, termed low level and high level, respectively (Table 1 ). These exposure conditions were scaled to match peak accelerations in an adult man, as previously described in detail [34-36]. The high acceleration level for a rabbit corresponded for an adult man to $11 \mathrm{krad} \cdot \mathrm{s}^{-2}$, considered sufficient to induce a moderate TBI $[25,37]$. The low exposure level was equivalent to $6 \mathrm{krad} \cdot \mathrm{s}^{-2}$ for an adult man, likely to cause a mild TBI, and of the same magnitude as the calculated average rotational acceleration of the head of professional American football players at concussions. Each animal was repeatedly exposed to either flexion or extension, or both, at low and high acceleration levels (Table 1). Data from previously reported extension experiments were included for comparison [34].

2.3. Animals and Exposure System. Adult New Zealand albino rabbits $(2.3-3 \mathrm{~kg})$ were kept anaesthetized throughout 


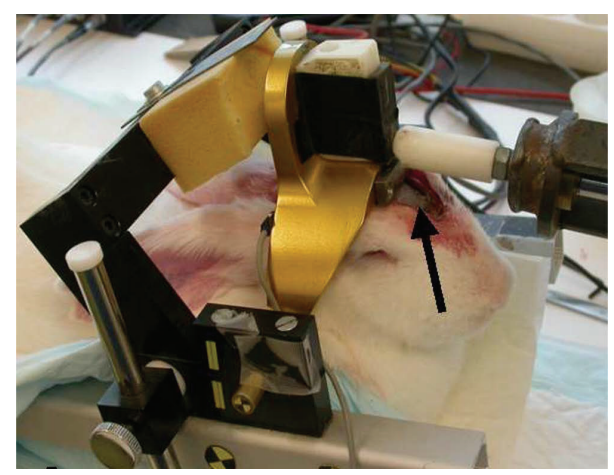

FIgure 1: The model system. The setup is shown with an anaesthetized rabbit in position for exposure to a closed head sagittal rotational acceleration. The arrow points to the glass fiber reinforced plastic helmet, glued to the cranium. A piston, attached to a pneumatic cylinder and driven by compressed air, was used to deliver the force to the moving (golden) arc to which the rabbit head was attached, as was an accelerometer.

the experiment by injections of Dormicum (Roche) and Hypnorm (Janssen). A helmet of glass fiber reinforced epoxy plastic was glued to the exposed calvarium, providing a broad-based, firm attachment between the rabbit head and a bar, which rotated around a horizontal axis at exposure (Figure 1) [34]. The animals were not restrained except for the attachment of the head. The position of the anaesthetized animals at flexion and extension in the exposure equipment only differed with regard to the orientation of the longitudinal axis [35]. The angular displacement of the head was for both flexion and extension set to $21^{\circ}$, based on pilot experiments, to limit mechanical deformation of the cervical spinal cord and the brainstem. The center of rotation was approximated to the center-of-gravity of the head, located inferior to the brain, and close to the pituitary [36] to minimize the risk for spinal cord damage. The bar, when struck by a piston driven by compressed air, forced the head to rotate in sagittal plane while the acceleration was recorded $[35,36]$.

Further, 3 animals were sham-exposed and 4 naïve controls used for comparison. In all, 42 animals were used in the present study.

2.4. Pressure Recording System. Pressures generated by the brain deformation were recorded with a fiber optical miniaturized pressure transducer (FOPT), connected to a control unit (Samba 3200; Samba Sensors AB, Sweden; Figure 2) and a computer. The FOPT was inserted in a $0.9 \mathrm{~mm}$ (outer diameter) polytetrafluoroethylene (PTFE) tube to protect the sensor from direct mechanical influence by the brain tissue. The pressure range was -0.8 to 5.5 bars, relative to the continuously recorded ambient atmospheric air pressure, and the average resolution 0.008 bar. Two pressure sensors were used concomitantly and supplied an analogue signal updated at $15 \mathrm{kHz}$ (filtered signal) and were acquired with the acceleration signal with a DataBRICK system (GMH Eng. Inc., Orem, UT, USA) at $12.8 \mathrm{kHz}$. No filters were applied to these signals to facilitate proper resolution in time. Aliasing was avoided for the pressure recordings as the oscillation frequency provided by the Samba unit was lower than the sampling frequency. The recorded accelerations were identical with an antialiasing filter set at $2500 \mathrm{~Hz}$ as compared to those without. System performance was checked prior to each test. The factory calibrated pressure sensors were additionally calibrated prior to and after use by immersion in a water-filled glass cylinder. Care was taken to keep the sensors clean.

2.5. Implantation of Pressure Transducers and Assessment of Structural Brain Injury. Two FOPTs, enclosed by PTFE tubes, were during each experiment inserted perpendicular to the rotation direction in the brain parenchyma through tightly fitting holes drilled in the skull [34]. The anterior FOPT, designated the parietotemporal one, was inserted through the parietal bone ( $8 \mathrm{~mm}$ lateral to the midline; $6 \mathrm{~mm}$ posterior to the bregma) and directed coronally toward the basal ganglia [38]. The posterior FOPT, named the occipital one, was positioned ipsilaterally through the occipital bone $6 \mathrm{~mm}$ behind the first one and directed towards the brainstem. The sensing tips were positioned $6-16 \mathrm{~mm}$ below the dura mater. Great care was taken to ensure that the sensors were positioned at the same locations during flexion as extension. The FOPTs were not sealed to the skull, except in one low level flexion experiment in which they were secured to the bone by dental glue. The FOPT cables were kept perpendicular to the rotation plane of the head. The FOPTs were in one set of experiments inserted at a 45-degree angle relative to the plane of rotation and thereby positioned close to each other, one angled rearward and the other angled forward. In other experiments, the two transducers were inserted in opposite brain hemisphere with the sensors facing each other. Holes were further drilled for recordings in the cerebellum.

Additional animals (Table $1 ; n=6$ ) were exposed to a low level sagittal rotational acceleration flexion impulse to investigate the function of the blood-brain barrier (BBB) 15 min after an intravenous injection of the marker Evans blue ( $1 \%$ in buffered saline; $1 \mathrm{~mL} / \mathrm{kg}$ b.w.) as the $\mathrm{BBB}$ is considered to remain essentially intact at a mild TBI. One $\mathrm{h}$ later, the animal was fixed by transcardial perfusion with buffered formalin after an initial rinsing with buffered saline. The brains were inspected macroscopically for extravasation of the dye-protein complex and thin sections prepared for fluorescence microscopy and examined for BBB dysfunction. Further animals $(n=5)$ had a single low level flexion exposure prior to fixation by transcardial perfusion to elucidate effects on blood vessels in the subarachnoid and VirchowRobin spaces. Sham-exposed $(n=3)$ and naïve control animals $(n=4)$ were investigated as well (Table 1$)$.

All animals were at the end of the experiments euthanized by overdose of Dormicum and Hypnorm. The FOPT tracks were inspected visually and with an operating microscope. The position of the sensor was thereby checked and named according to a rabbit brain atlas [38].

2.6. Analysis of Pressure Recordings. Differences in peak pressures (positive peak pressures recorded in extension trauma 

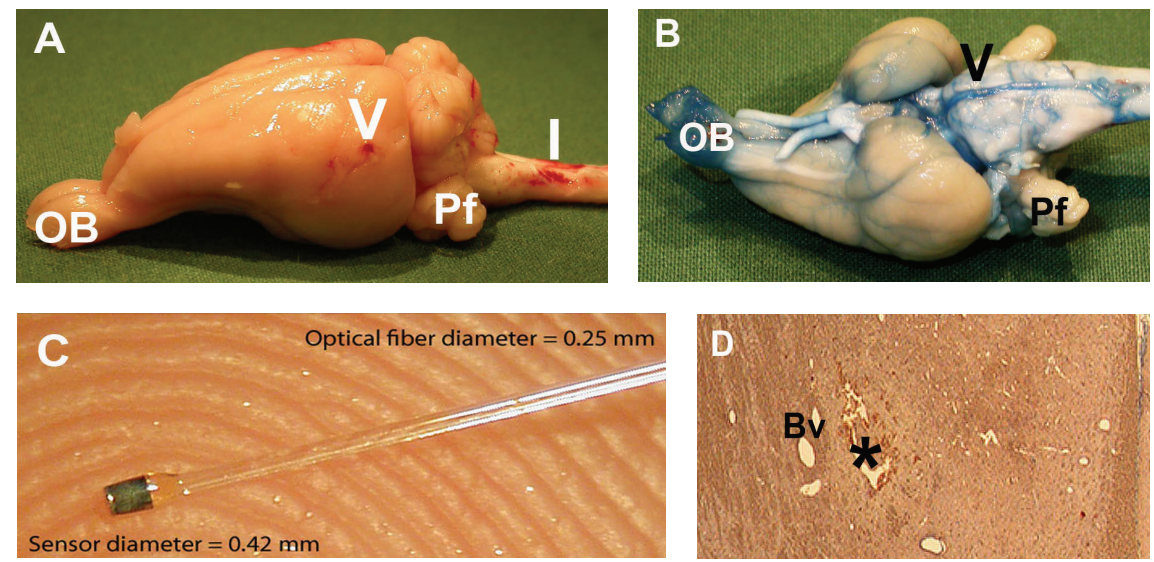

FIGURE 2: Effects on brain by exposures. A rabbit brain after a single exposure to a low level sagittal flexion closed head rotational acceleration (A). There are small hemorrhages in the leptomeninges and on the dorsal surface of the medulla and uppermost cervical cord (marked I). The track after a FOPT used in this special exposure indicated by an arrowhead. Paraflocculus (Pf) and the olfactory bulbs (OB) marked. The brain has no signs of contusion or distortion. (B) shows that there was no blood-brain barrier dysfunction but in the olfactory bulbs (OB), as revealed by Evans blue staining after a low level flexion exposure. The arrowhead points to a stained segment of the basilar artery. Note the staining along the vessels in the subarachnoid spaces. (C) shows the design of a FOPT, resting on a fingertip. The sensor is to the left at the tip of the glass fiber conducting LED light. Note the small dimensions and the absence of mechanical parts and electric leads. (D) Light micrograph of a stained section of brain parenchyma with a tiny hole $(*)$ constituting the track formed by a FOPT after two exposures to a high level extension. Blood vessel marked Bv.

and negative peak pressure recorded in flexion trauma) between flexion and extension trauma for measurements in one region of the brain and between different measurement sites for different trauma levels were analysed using a one-way analysis of variance (ANOVA) blocked by sample and a post hoc test at a 5\% significance level. All differences analysed with aforementioned statistical methods were assessed for the first peak; the second peak that appeared in some extension tests was neglected.

\section{Results}

3.1. Macroscopically Visible Injuries. Rabbits exposed to either a low level flexion or low or high level extension sagittal rotational acceleration, closed head impulse had no skeletal fractures or dislocations; all breathed spontaneously and none had any apnea (all survived). In contrast, all rabbits exposed to a high level flexion suffered lasting apnea and died at the exposure but had neither any skeletal fractures or dislocations nor any deformation of the head and neck. The carotids and their main branches and the vertebral arteries were inspected and found to be intact in all exposed animals. Sham-exposed animals were unaffected as were the naive controls.

Autopsy of three rabbits that succumbed at the exposure to a single high level flexion disclosed scattered parenchymal and extensive meningeal hemorrhages, extending to the cervical spinal cord, but no skeletal fractures, contusions, lacerations, or torn structures. In contrast, animals exposed to a low level flexion rarely showed rupture of cerebral cortical veins, thin hemorrhages in the leptomeninges and along Virchow-Robins spaces, and leptomeningeal hemorrhages at the transition between the cervical spinal cord and the brainstem (Figure $2 \mathrm{~A}$ ). No focal injuries or hemorrhages were demonstrable in the brain parenchyma, not even underlying the helmet or at the countercoup position. The $\mathrm{C} 1$ and occasionally the $\mathrm{C} 2$ dorsal roots were teased but never ruptured. The spinal cord appeared macroscopically intact. The olfactory bulbs were partly detached from the cribriform plates and petechiae recognized. The BBB was as expected at a mild TBI intact except for regarding the olfactory bulbs (Figure 2 B). However, the leptomeninges looked slightly blue as did parts of the basilar artery and some leptomeningeal vessels, most evidently in the subarachnoid and VirchowRobins spaces, indicating slight dysfunction of the vascular barriers but without prevalence of hemorrhages or meningeal ruptures.

Brains from rabbits exposed to a closed head extension trauma at either low or high level looked at autopsy like those from sham-exposed animals and naive controls, that is, macroscopically uninjured.

Small hemorrhages were observed in immediate connection to and along the tracks formed by the FOPTs, especially after multiple runs (C and D in Figure 2).

3.2. Pressure Measurements at Head Flexion. At a low level flexion rotational acceleration, negative pressures (also named underpressures in this report and related to ambient air pressure) dominated (Figures 3(a) and 3(b); Table 1). The acceleration recordings are shown in Figures 3(a) and 3(c). The posterior FOPT, positioned in the upper brainstem, showed an initial deep pressure drop lasting approximately $2 \mathrm{~ms}$, sometimes followed by a transient positive pressure (also named overpressure and related to ambient air pressure). The anterior FOPT, positioned in the basal ganglia, similarly disclosed an initial pressure drop lasting about $2 \mathrm{~ms}$ 


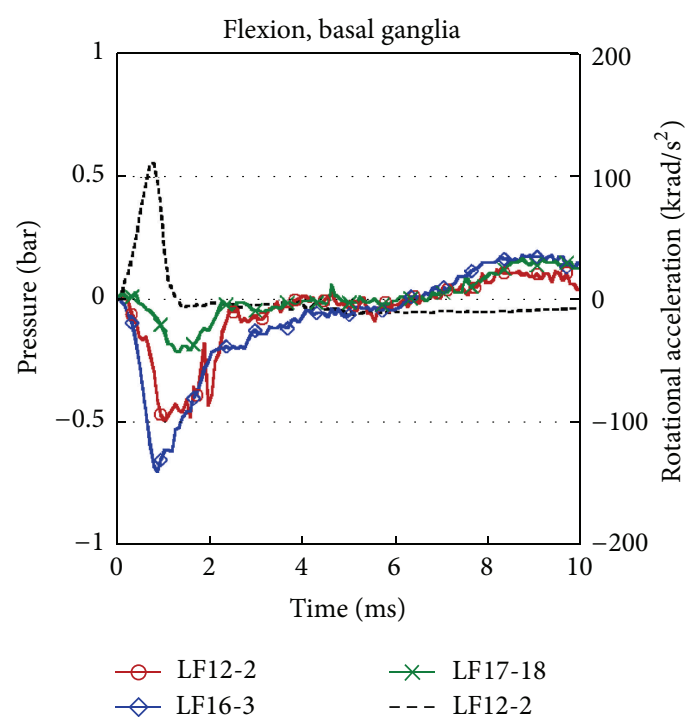

(a)

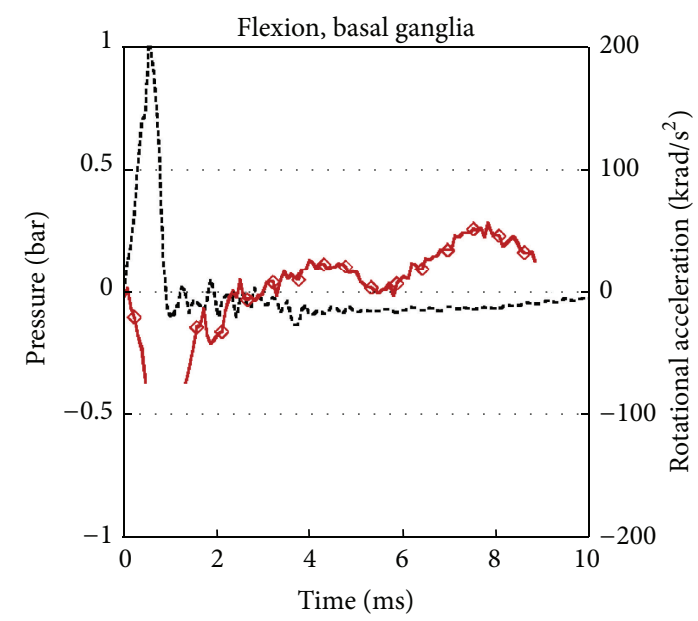

$\diamond$ HF12-1

- - - HF12-1

(c)

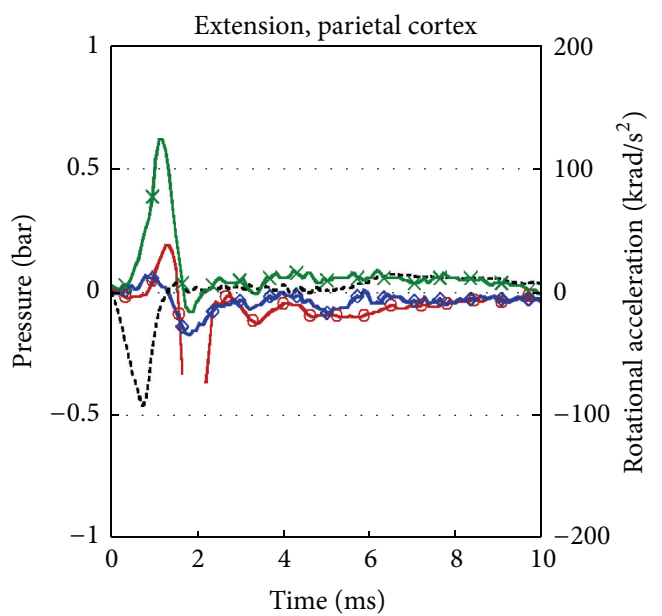
- $\mathrm{LE} 3-5$
$\leftarrow$ LE6-5
- - - LE5-3

(e)

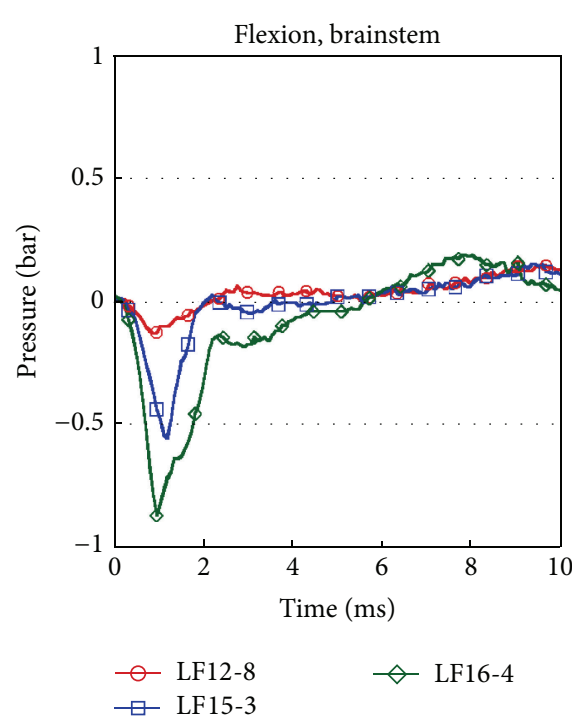

(b)

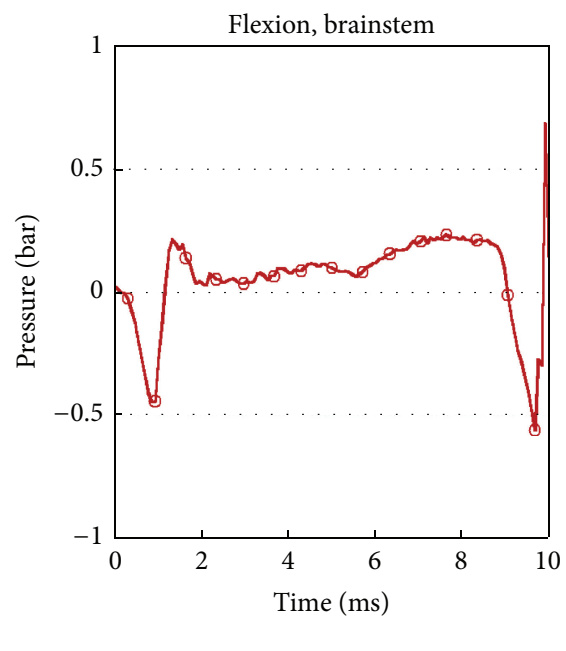

- HF12-1

(d)

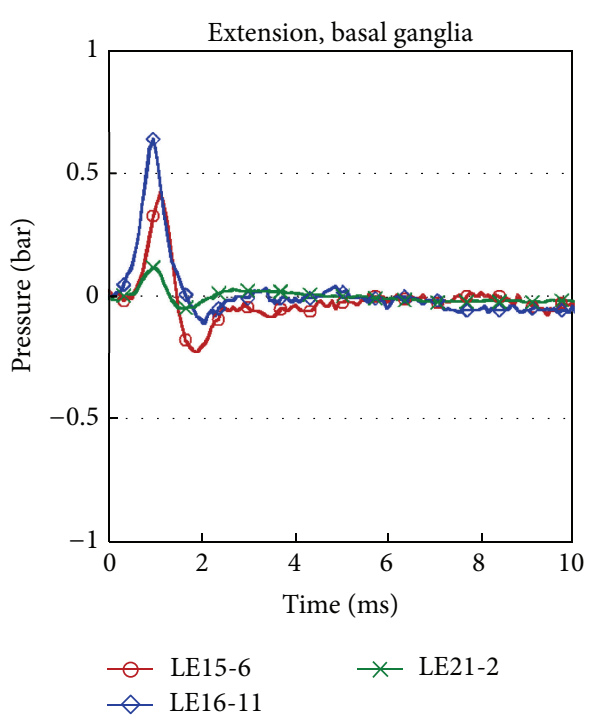

(f)

Figure 3: Continued. 


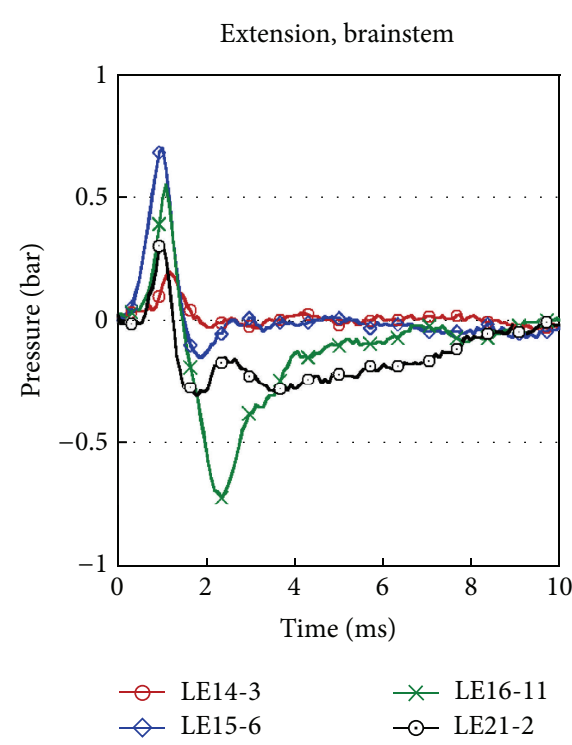

(g)

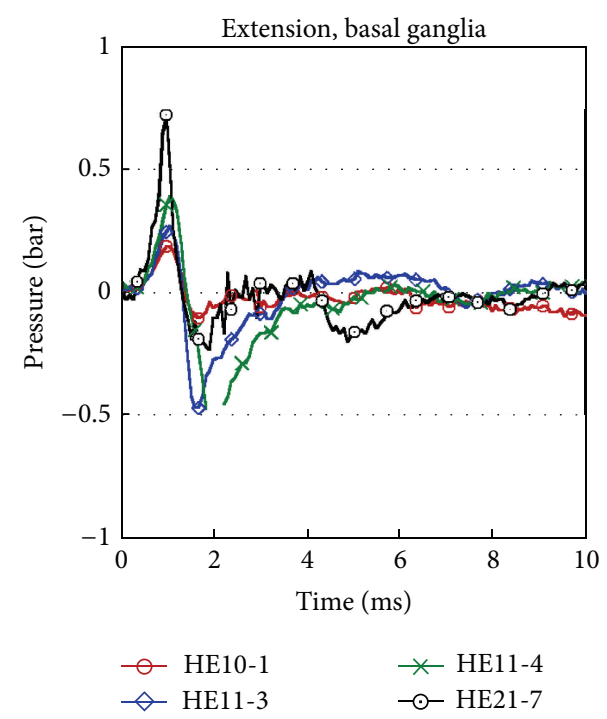

(i)

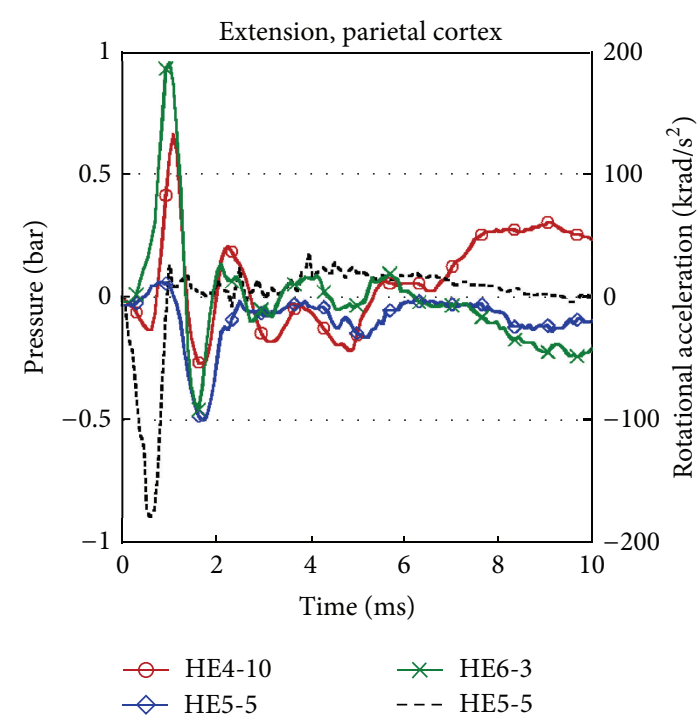

(h)

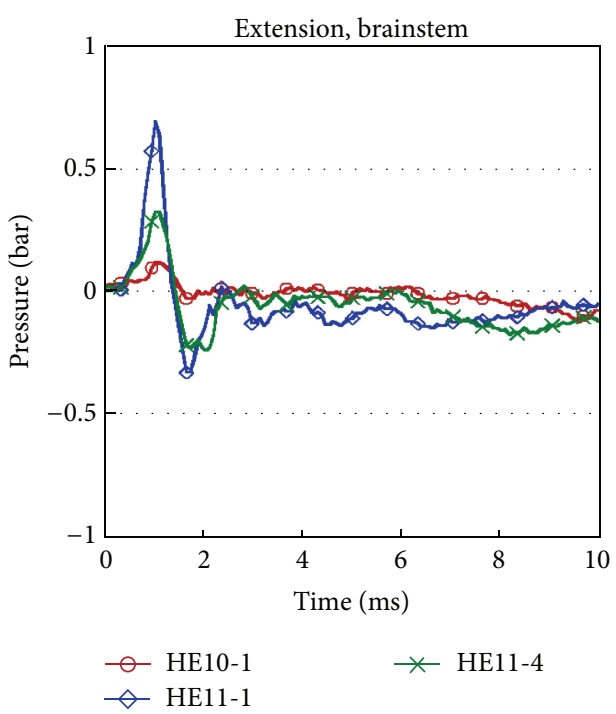

(j)

FIGURE 3: Sagittal closed head rotational acceleration and pressure recordings in indicated regions of the brain during low and high level flexion $((a)-(d))$ and extension $((e)-(j))$, respectively. The recorded sagittal rotational acceleration rate is indicated by the black dashed line and the scale to the right in (a), (c), (e), and (h). (a) and (b) demonstrate that a low level flexion initially induced a strong underpressure, followed by slight overpressure. (c) and (d) show the strong pressures recorded at a high level flexion. Extension at a low level ((e)-(g)) generated initially overpressure followed by underpressure, thus strikingly differing from the pressure patterns induced by flexion at the same level ((a) and (b)). Pressure patterns at high level extensions are presented in (h)-(j). Three experiments were performed in 3 different animals, except for high level flexion. The largest change in peak pressures, the least change in pressure, and representative pressure time histories are presented. See Figure 8 for statistical analyses.

and then ambient pressure, sometimes after a transient, slight overpressure.

All animals exposed to a high level flexion sagittal rotational acceleration impulse died at the exposure. The pressures mostly exceeded the sensors measuring range (Figures 3(c) and 3(d); Table 1).

Consecutive low level flexion exposures in the same animal showed acceptable pressure repeatability (Figures $4(\mathrm{a})-4(\mathrm{~d}))$. The recorded negative peak pressure varied when all tests were studied (Figures 3 and 4), but the pressure patterns were similar, indicating reproducibility.

Implantation of two pressure sensors just a few $\mathrm{mm}$ apart at the same depth in the brain parenchyma resulted in small differences in the pressure patterns.

A sensor in the paraflocculus of the cerebellum disclosed that a low level flexion induced positive and negative pressure peaks divergent from those concomitantly recorded by a second sensor in the brainstem (Figures 5(a) and 5(b)). 


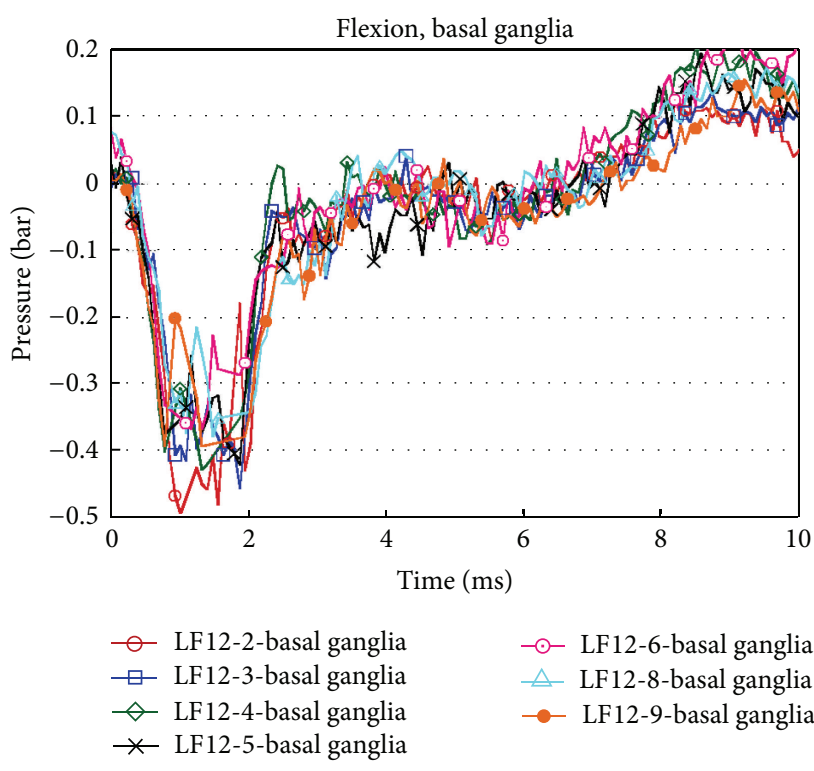

(a)

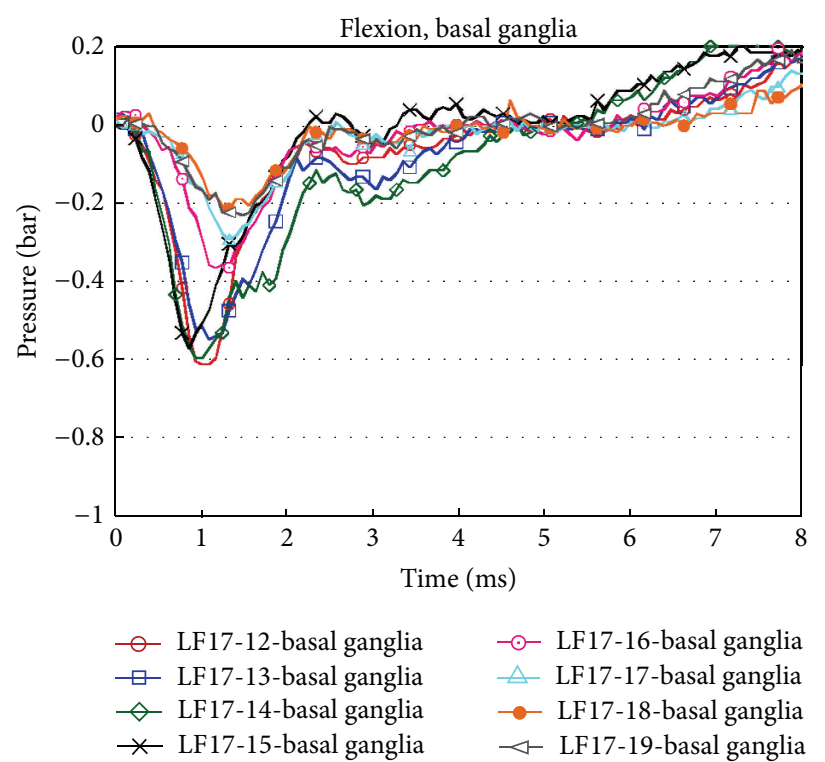

(c)

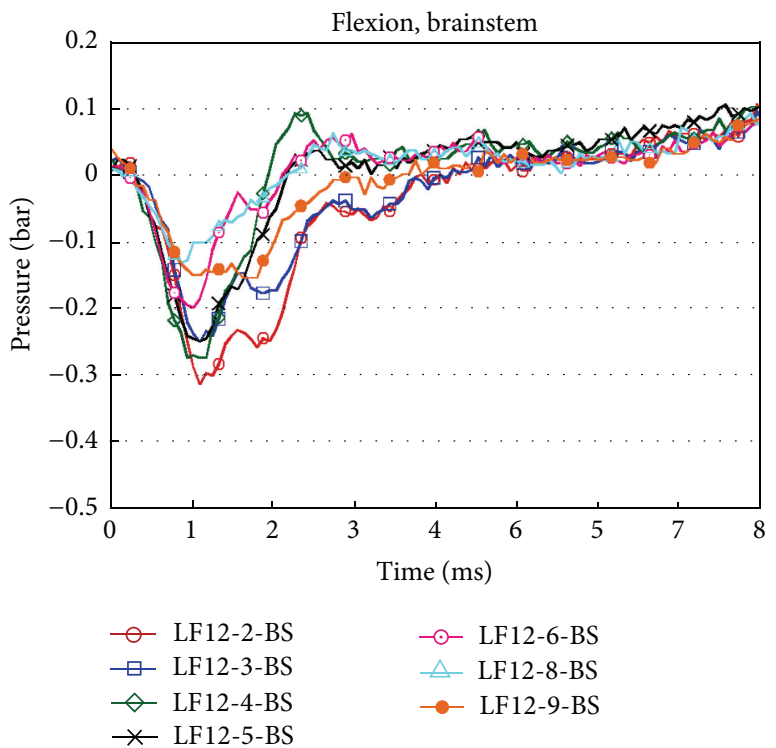

(b)

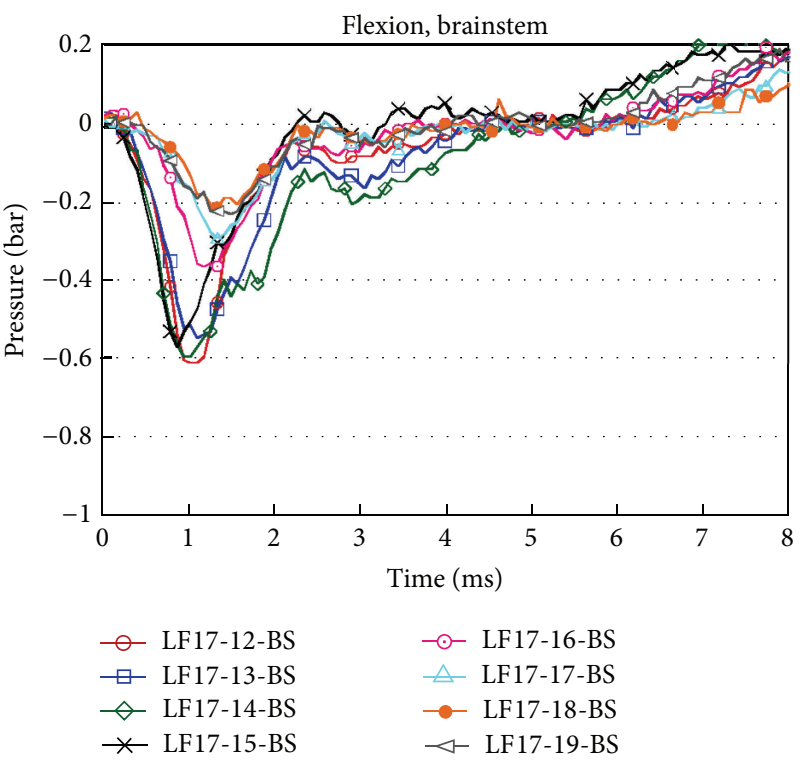

(d)

FigURE 4: Repeated pressure recordings in animal number 12 ((a), (b)) and animal number 17 ((c), (d)) with sensors kept at the same locations in the brain parenchyma throughout a series of repeated consecutive low level sagittal plane flexion rotational acceleration exposures. Recordings from the basal ganglia (left) and the brainstem (BS; right). Close to the same pressure, patterns are recorded, but larger amplitudes are obtained at the first exposures. See Figure 8 for statistical analyses.

An initial positive peak was recorded by the paraflocculus sensor and followed by a negative peak. There was $\sim 0.5 \mathrm{~ms}$ time difference between the two sensors as the pressure changes occurred later in the paraflocculus than in the brainstem (Figure 5(a)). A sensor deep in the cerebellum recorded at a low level flexion a pressure pattern similar to that in paraflocculus (Figure 5(b)). A time delay was consistently demonstrable between the pressures in the different parts of the cerebellum as compared to those in the cerebrum and brainstem.
3.3. Pressure Measurements at Head Extension. At low level extension, an initial positive pressure peak was followed by a brief negative one (Figures 3(e)-3(j)). The accelerometer readings are shown in Figures 3(e) and 3(h). These recordings were similar to those previously reported [34]. For a high level extension, the pressure drop was more prominent in the basal ganglia as compared to that in the rostral brainstem (Figures 3(f), 3(g), and 5(c)).

A sensor inserted in the border between the pons and medulla oblongata, that is, in caudal parts of the brainstem, at 


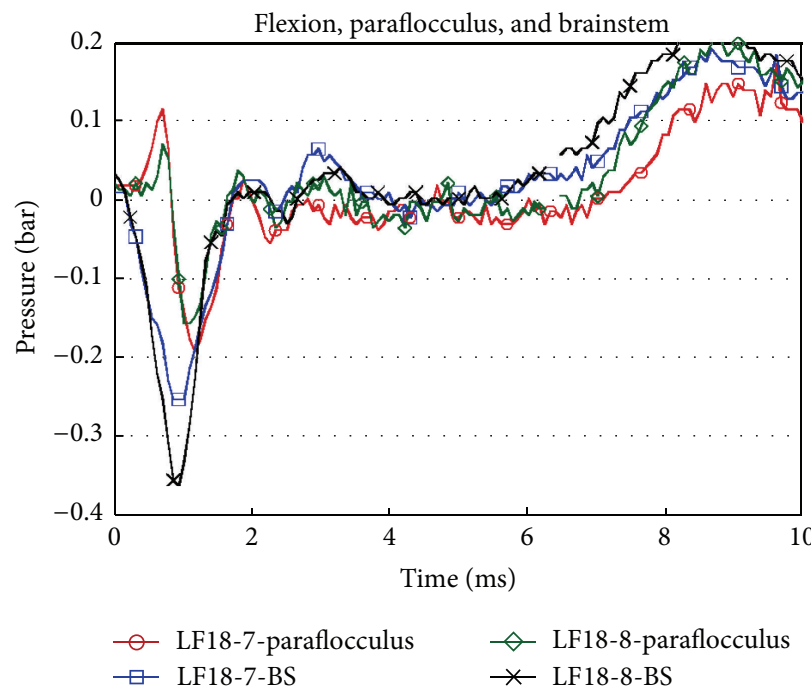

(a)

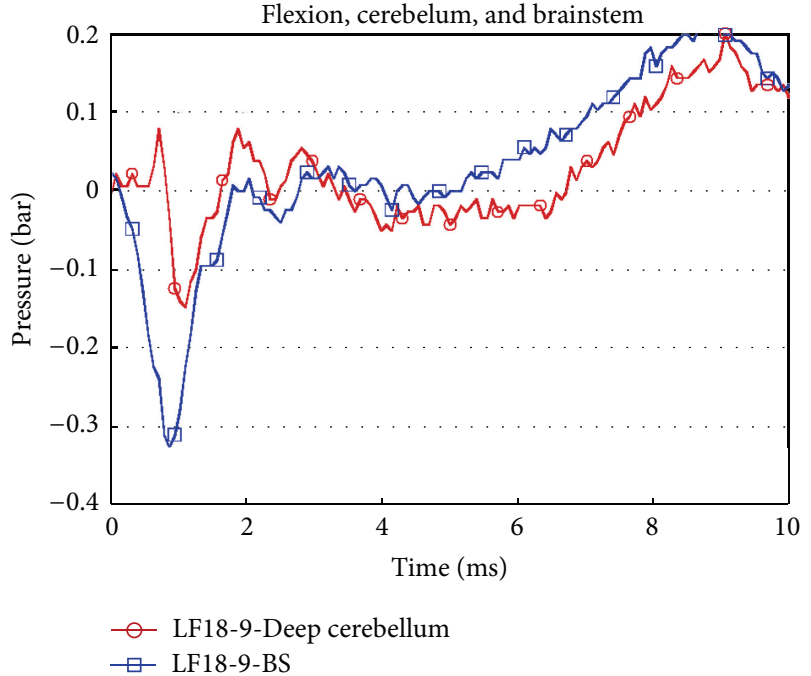

(b)

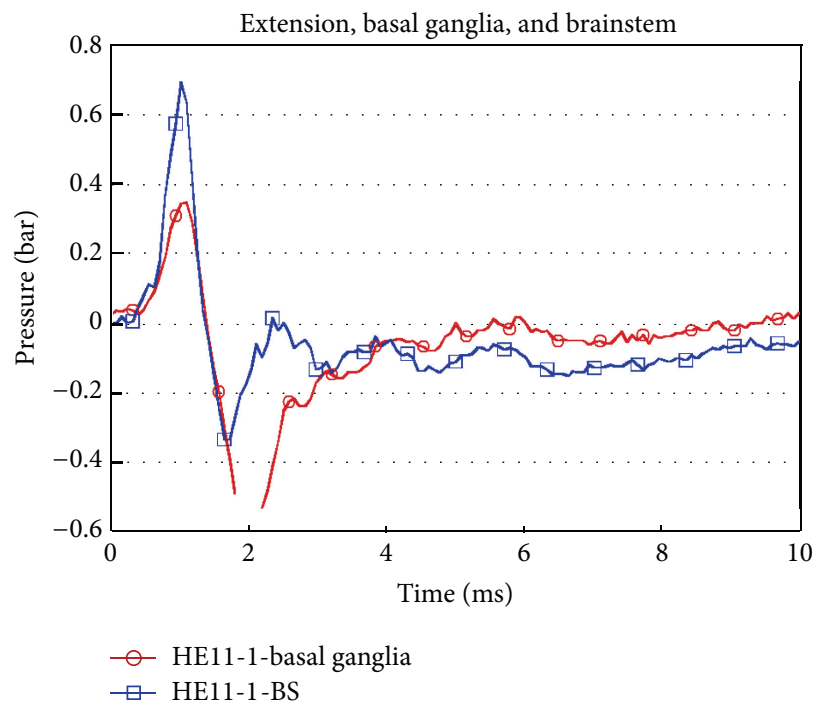

(c)

FIGURE 5: Two consecutive pressure recordings in the same animal at two locations, brainstem (BS) and paraflocculus cerebelli (a), and a subsequent one with one sensor in the brainstem and the second one deep in the cerebellum (b) at a low level sagittal plane flexion rotational acceleration. Note the difference in time between the appearance of pressure changes in the brainstem and in the cerebellum. A high level sagittal extension trauma (c) induced strong pressure amplitudes, initially overpressure followed by underpressure, with one sensor positioned in deep in the caudate head (basal ganglia) and the other one in the pons portion of the brainstem. The sensor in the brainstem indicates extended, persistent underpressure due to extensive deformation.

a high level extension closed head exposure recorded a strong overpressure followed by underpressure, while the anterior FOPT, placed in the basal ganglia, revealed an even stronger pressure drop (Figure 5(c)). The pons sensor recorded a sustained negative pressure for a prolonged time as compared with the sensor in the caudate head of the basal ganglia (Figure 5(c)).

3.4. Pressure Measurements to Elucidate the Importance of Direction and Anchoring of the Sensors. Two sensors, implanted in mirror positions in either brain hemisphere, revealed similar pressure patterns at both flexion and extension rotational accelerations (Figures 6(a) and 6(b)) with small differences, likely due to variations in sensor positions.

Attaching the PTFE tube to the skull bone with glue had no measureable effects on the pressure recordings at exposure to low level flexion, in concordance with our previous report on extension [34].

The insertion of the sensors in deep brain structures at an angle of approximately 45 degrees relative to the plane of rotation, with the anterior FOPT angled in a posterior direction and the posterior one angled anteriorly, had little or no effect on the recorded pressures (Figures $7(a)$ and $7(b)$ ). 


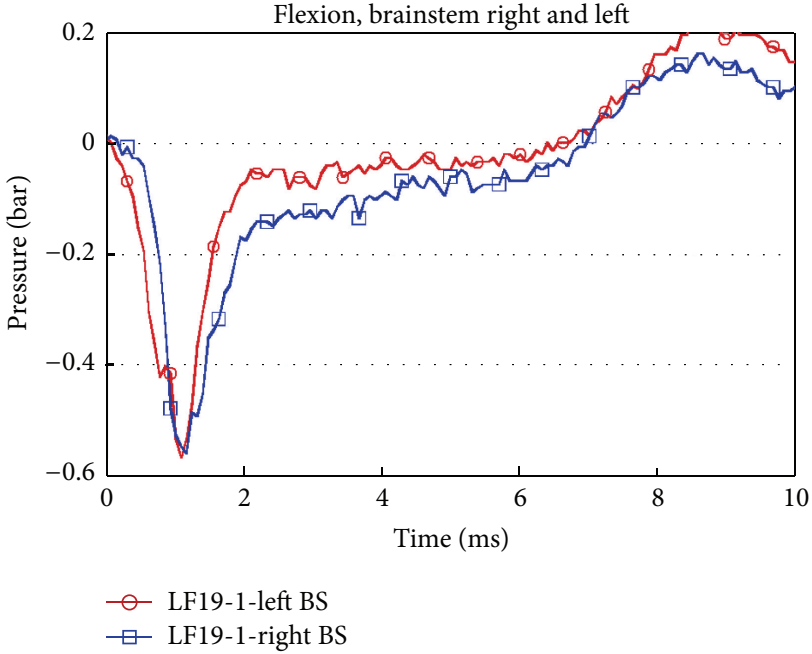

(a)

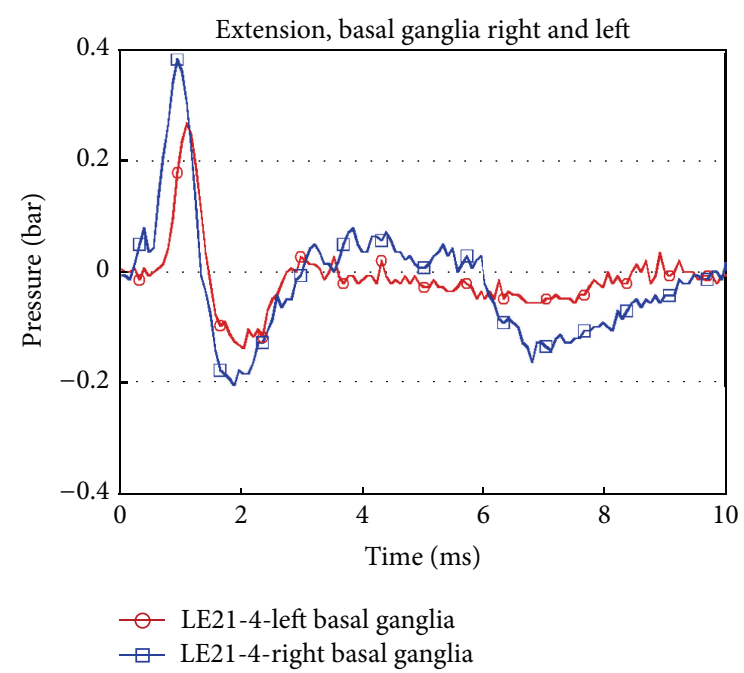

(b)

Figure 6: Two FOPTs in opposite brain hemispheres from the right and the left side, facing each other, recorded the pressure in the left or right side of the brainstem (BS) at a low level flexion (a) and basal ganglia at a low level extension (b). Similar pressure recordings were obtained for both the brainstem and the basal ganglia, respectively, at either exposure direction. The variations reflect minor differences in sensor positions.

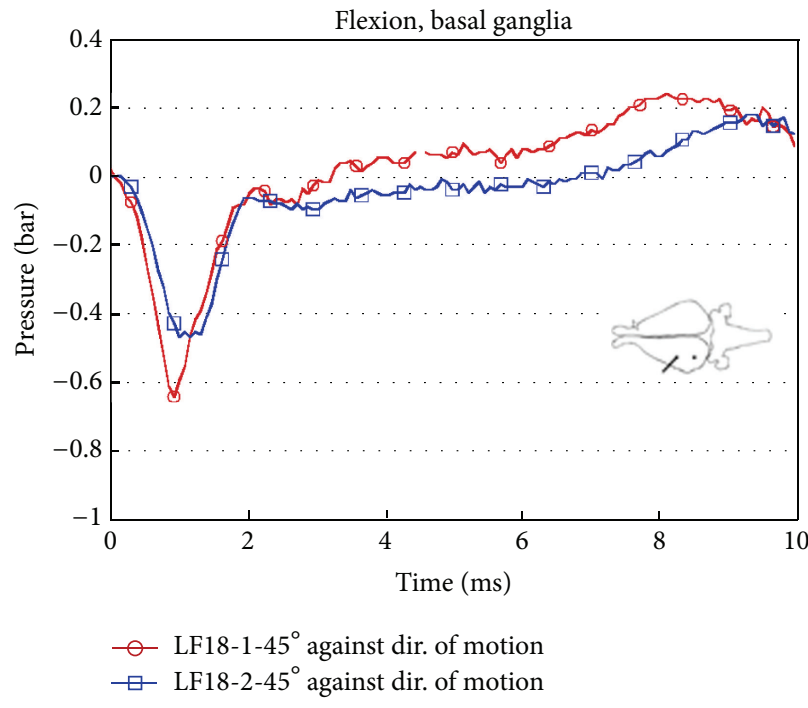

(a)

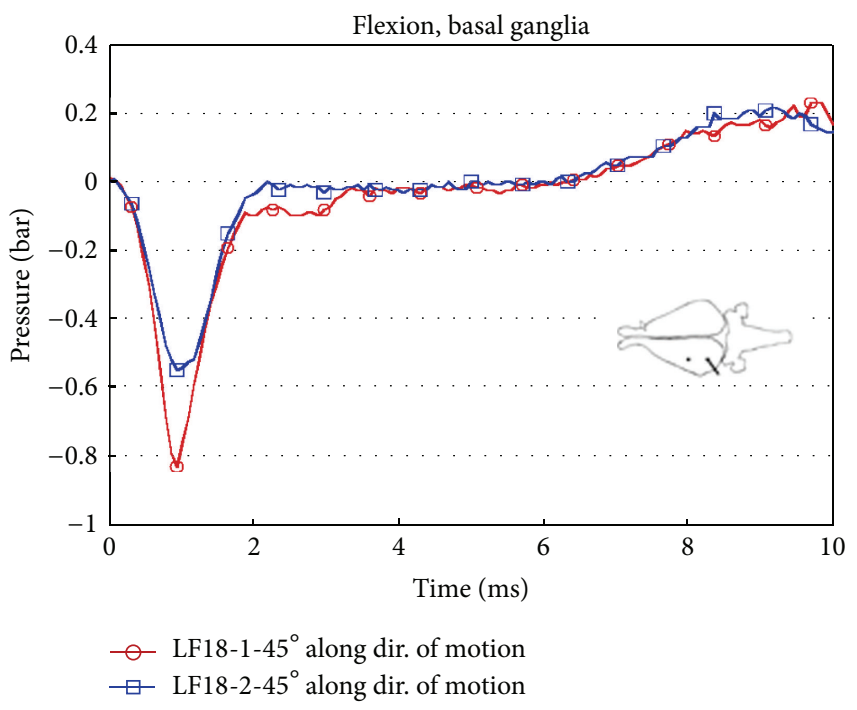

(b)

FIGURE 7: Repeated recordings at a low level sagittal flexion closed head rotational acceleration with the sensors in basal ganglia. One FOPT was inserted through the hole in the parietotemporal bone and directed at an angle of $45^{\circ}$ rearward (a), while the one through the occipital bone was directed at an angle of $45^{\circ}$ forward (b). Thereby the sensor tips were positioned only a few mm apart during the exposure. Similar pressure patterns were obtained whether the sensor was aligned against or along the direction of the sagittal rotational acceleration. The sensors are thus likely to record dynamic pressures generated in the brain parenchyma at the exposure. No obvious indication suggesting that the pressures were related to movements of the FOPTs in the brain tissue.

3.5. Assessment of Structural Brain Injury Caused by the Two FOPTs and by the Exposure. The tracks formed by the FOPTs were traced visually and by light microscopy to map the positions of the transducers. Light microscopy of stained brain sections disclosed that a FOPT track generated a collapsed channel (Figure 2 D). Hemorrhages and tissue contusions were rarely observed after a single exposure but after multiple exposures and when the FOPT position was changed.

In a published report, we have characterized the histopathological changes demonstrable in exposed animals surviving one week after an exposure to sagittal plane rotational acceleration impulse as described above. Diffuse brain injuries were demonstrable in the brains of rabbits exposed 
to a sagittal plane low level flexion rotational acceleration impulse as could be shown for animals exposed to high or low level extension [35]. The animals exposed to a high level flexion suffered brain contusions and none survived, and therefore no corresponding histopathological data are available. These histopathological investigations on animals exposed to a low level flexion and a high or low level extension thus demonstrate that the used exposure levels are to be considered causing mild TBI according to commonly used criteria $[10,18,20,22,39]$.

3.6. Statistical Analysis of the Results. The statistical analysis of the achieved results is presented in Figure 8.

\section{Discussion}

A major result was that strikingly differing pressure patterns were generated in the brain depending on the direction of movement of a head at a sagittal closed head rotational acceleration impulse and equal force lasting close to $1 \mathrm{~ms}$ [Figure 8]. Flexion resulted in pressures with longer duration and larger amplitudes than did extension. A low level flexion induced prominent negative, prolonged pressures in contrast to extension, which resulted in a short positive pressure peak followed by a small negative one (Figure 3). Pudenz and Shelden [28] reported that the brain moves and may be deformed at a closed head trauma, as subsequently repeatedly confirmed $[6,7,10,17,29,30]$. The recorded pressures were generated by deformation of the brain and spinal cord on exposure to a sagittal plane rotational acceleration. The pressure pattern and its duration constitute a measure of the inertial twisting and strain at the deformation of the brain. We conclude that flexion caused more extensive mechanical trauma to the brain than did extension at the same force, in agreement with what has previously been demonstrated histopathologically [35]. At a low level flexion, there were macroscopically no visible hemorrhages, contusions, or tearing of the brain parenchyma and spinal cord, but histopathologically demonstrable diffuse brain injuries [35]. Consistent findings were mechanical damage to the olfactory bulbs, hemorrhages in the leptomeninges and along the Virchow-Robins space, and deformation of the $\mathrm{Cl} / \mathrm{C} 2$ nerve roots (Figures 1 and 2). The exposure to flexion thus likely overstretched the brain and spinal cord. Unexpectedly, we did not observe any apnoea, considered a sensitive marker of brainstem damage, except at a high level flexion. The paraflocculus, which in rabbits is largely enclosed by bone, showed the same pressure pattern as deep central parts of the cerebellum (Figure 5). The cerebellum was histopathologically damaged but less than the olfactory bulbs [35], which in parts detached from their normal anchoring to the cribriform plates. Extension, similarly limited to $21^{\circ}$, likely compressed the brain but caused no macroscopic signs of damage [34, 35]. The prevalence, distribution, and severity of brain and spinal injuries are known from other types of exposures to be direction dependent $[6,7,9,10,15,24,27]$. Our published histopathological investigations have disclosed that flexion caused much more extensive damage than did extension

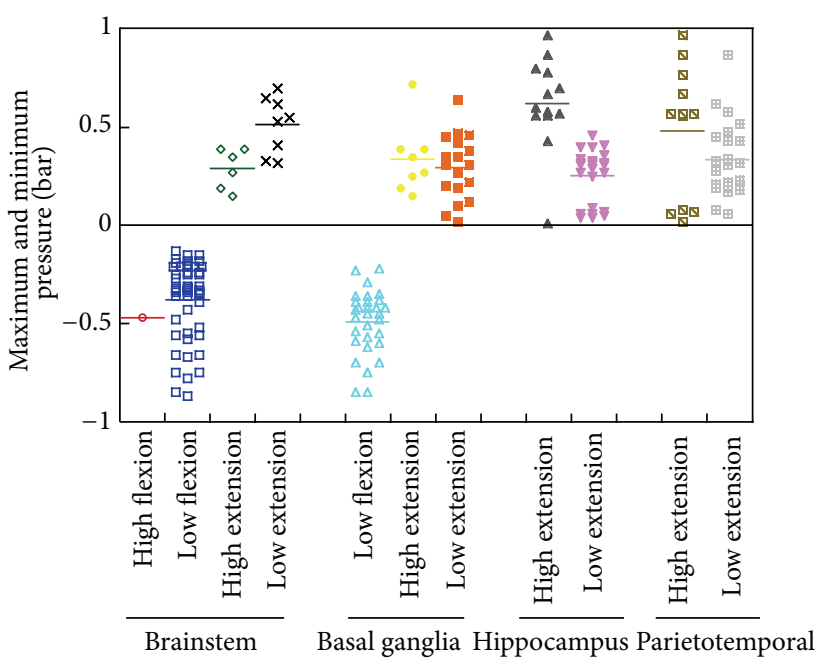

FIGURE 8: The ANOVA of the pressure data provided the following results: for pressure measurements in the brainstem: low flexion was significantly different from low extension. Pressure in the brainstem was not significantly different between low and high extension. For pressure measurements in the basal ganglia: low flexion was significantly different from low extension. Pressure in the basal ganglia was not significantly different between low and high extension. For pressure measurements in the hippocampus: low extension was significantly different from high extension. For pressure measurements in the parietotemporal cortex: low extension was not significantly different from high extension. For pressure measurements in low extension trauma: only the peak pressure in the hippocampus was significantly different from the peak pressure in the brainstem. All other combinations of pressure recordings in the brainstem, basal ganglia, hippocampus, and parietotemporal cortex were found not to be significantly different from each other when exposed to a low extension trauma. For pressure measurements in high extension trauma: only the peak pressure in the hippocampus was significantly different from the peak pressure in the brainstem and basal ganglia. All other combinations of pressure recordings in the brainstem, basal ganglia, hippocampus, and parietotemporal cortex were found not significantly different from each other when exposed to a high extension trauma. For pressure measurements in low flexion trauma: peak pressures in the brainstem and basal ganglia regions were found not to be significantly different from each other.

at the same force [35]. It must however be stressed that in either case the diffuse brain injury demonstrated must be classified as fulfilling the criteria for being mild TBI as there was no evidence indicating any contusion, tearing, or parenchymal hemorrhages. We conclude that the increased magnitude and duration of the recorded transient pressures at flexion as compared to extension movements in the brain parenchyma correlated with the macroscopically and histopathologically more extensive brain damage [35]. Case reports in the forensic medicine indicate that flexion may be more deleterious than extension also to human subjects, but to our knowledge no systematic investigation is available $[20,22]$.

Our presented results elucidated the importance of rapid, transient vibrations and movements of a head at impact for 
the severity of the resulting brain damage. In our initial experiments [36], the force delivering helmet was attached to the cranium with individually glued titanium pins, which enabled movements of the cranium and thus of the head as less than $40 \%$ of the calvaria was anchored to the helmet. In contrast, in the present experiments, the entire calvaria was firmly attached with glue to a fitting helmet [34], restricting head movements. Further, the glue formed a continuous layer firmly bridging the calvarial bone and the shaped helmet of glass fiber reinforced epoxy, thereby minimizing any mechanical deformation of the skull. The head movements allowed by our previous system strikingly increased the severity of the damage as reflected by, for example, brain hemorrhages, widespread diffuse brain injury, elevated levels of excitotoxic amino acids, and extensive gliosis [36, 40, 41]. At simulated traffic accidents, brain damage may be avoided if head and neck movements are limited [14]. We conclude that restricting the head movements and deformation of the skull, as done in the present study, reduced the extent and severity of the induced TBI [35], in agreement with considerations in clinical and experimental reports [14, 20, 22, 28, 42].

We asked whether the recorded transitory pressure changes at a rotational acceleration, closed head impulse could add to the injury in the anisotropic brain. Support is gained from studies based on finite element modeling and blast-induced TBI at pressures of similar magnitude and duration [19, 43-47]. Distant high energy missile hind leg impact to animals induces positive and negative pressure waves in the brain as well as histopathologically demonstrable brain damage [48]. Pressure waves of similar magnitude and duration are injurious to cultured neurons [49]. Detailed mapping of the deformation and pressure patterns at a sagittal rotational acceleration is required to derive firm statements about whether the induced pressure waves per se added to the diffuse brain injury.

The brain lacks conventional lymphatics [50-54]. Instead, the cerebrospinal fluid (CSF) and the interstitial (extracellular) fluid (ISF) are drained along paravascular pathways and nerves. It is essential for the brain to have a proper turnover of fluid, nutrients, and waste products. A main pathway for the ISF flow constitutes the VirchowRobin space (VRS) and further along brain blood vessels, draining fluid eventually to, for example, cervical lymph nodes. A closed head sagittal rotational acceleration trauma as in the present study resulted in mechanical displacement and deformation of the brain, including blood vessels and meninges, which may impair the blood flow and the traffic of CSF and ISF. Rabbits exposed to a flexion impulse had hemorrhages preferentially localized perivascularly in the subarachnoid space (A and B in Figure 2) and along the VRS. Histopathological investigation of exposed, surviving animals disclosed posttraumatic reactive gliosis along cortical arteries in the VRS [35]. In our opinion, a closed head trauma not only deforms the brain but in addition may impair the trafficking of fluid, metabolites, and waste products by paravascular routes, adding to the primary brain injury.

One limitation is that the achieved results are not readily applicable to humans, whose neuraxis has a sharp angle between the main part of the brain and the spinal cord while the neuraxis in rabbits is slightly S-shaped, as judged by the position at the exposures in the present study. Further, the human cerebrum is gyrencephalic, having distinct sulci and gyri, while the rabbit brain is lissencephalic, that is, smooth surfaced, likely to influence the outcome [51, 55]. However, the cerebellum both in humans and in animals has an elaborated system of fissures and folia which are injured more often than generally anticipated both in humans and in animals [22, 30, 35, 39].

\section{Conclusions}

Our approach to use the dynamic pressure patterns at a sagittal plane rotational acceleration trauma enables evaluation of the induced deformation of the brain. The information achieved with our approach supplements other available methods in assessing the movements and structural alterations going on in the brain at the exposure to a closed head trauma at a very high time resolution. Further, we could demonstrate the prevalence of time differences in displacement and deformation of different parts of the brain, as were revealed by the timing of the pressure patterns. We further conclude that a closed head sagittal flexion rotational acceleration impulse caused more extensive deformation of the brain and spinal cord than extension, generating stronger pressures of longer duration and aggravation of the induced TBI. Different parts of the brain are displaced and deformed in a noncoherent manner, which likely explain differences in extent of the resulting TBI. The events in the leptomeninges and Virchow-Robin spaces deserve elucidation with regard to likely importance for the generation of primary and secondary brain damages.

\section{Conflict of Interests}

The authors declare that they have no conflict of interests regarding the publication of this paper. Svante Höjer was initially an employee of Samba Sensor AB, but subsequently working at the University of Gothenburg.

\section{Acknowledgments}

This work was supported by grants from VINNOVA, SAFER, the W. and M. Lundgren Foundation, Lantmännen ASFaktor AB, Gothenburg Medical Society, the Swedish Federal Government, and the Sahlgren's University Hospital. The authors thank Per Lövsund and Margareta Rosenkvist for support, advice, and help.

\section{References}

[1] J. A. Langlois Orman, J. F. Kraus, E. Zaloshnja, and T. Miller, "Epidemiology," in Textbook of Traumatic Brain Injury, J. M. Silver, T. W. Mcalister, and S. C. Yudofsky, Eds., pp. 3-22, American Psychiatric Publishing Inc, Washington, DC, USA, 2nd edition, 2011. 
[2] V. G. Coronado, L. Xu, S. V. Basaravaju et al., "Surveillance for traumatic brain injury-related deaths-United States, 19972007," Morbidity and Mortality Weekly Report-Surveillance Summaries, vol. 60, no. 5, pp. 1-32, 2011.

[3] V. L. Feigin, A. Theadom, S. Barker-Collo et al., "Incidence of traumatic brain injury in New Zealand: a population-based study," The Lancet Neurology, vol. 12, no. 1, pp. 53-64, 2013.

[4] F. Tagliaferri, C. Compagnone, M. Korsic, F. Servadei, and J. Kraus, "A systematic review of brain injury epidemiology in Europe," Acta Neurochirurgica, vol. 148, no. 3, pp. 255-267, 2006.

[5] A. Kay and G. Teasdale, "Head injury in the United Kingdom," World Journal of Surgery, vol. 25, no. 9, pp. 1210-1220, 2001.

[6] W. N. Hardy, C. D. Foster, M. J. Mason, K. H. Yang, A. I. King, and S. Tashman, "Investigation of head injury mechanisms using neutral density technology and high-speed biplanar Xray," Stapp Car Crash Journal, vol. 45, pp. 337-368, 2001.

[7] W. N. Hardy, M. J. Mason, C. D. Foster et al., "A study of the response of the human cadaver head to impact," Stapp Car Crash Journal, vol. 51, pp. 17-80, 2007.

[8] E. D. Bigler and W. L. Maxwell, "Neuropathology of mild traumatic brain injury: relationship to neuroimaging findings," Brain Imaging and Behavior, vol. 6, no. 2, pp. 108-136, 2012.

[9] A. K. Ommaya, W. Goldsmith, and L. Thibault, "Biomechanics and neuropathology of adult and paediatric head injury," British Journal of Neurosurgery, vol. 16, no. 3, pp. 220-242, 2002.

[10] P. Reilly and R. Bullock, Head Injury-Pathophysiology and Management, Hodder Arnold, London, UK, 2nd edition, 2005.

[11] A. A. Sabet, E. Christoforou, B. Zatlin, G. M. Genin, and P. V. Bayly, "Deformation of the human brain induced by mild angular head acceleration," Journal of Biomechanics, vol. 41, no. 2, pp. 307-315, 2008.

[12] D. F. Meaney and D. H. Smith, "Biomechanics of concussion," Clinics in Sports Medicine, vol. 30, no. 1, pp. 19-31, 2011.

[13] M. E. Shenton, H. M. Hamoda, J. S. Schneiderman et al., "A review of magnetic resonance imaging and diffusion tensor imaging findings in mild traumatic brain injury," Brain Imaging and Behavior, vol. 6, no. 2, pp. 137-192, 2012.

[14] D. R. Namjoshi, C. Good, W. H. Cheng et al., "Towards clinical management of traumatic brain injury: a review of models and mechanisms from a biomechanical perspective," Disease Models \& Mechanisms, vol. 6, no. 6, pp. 1325-1338, 2013.

[15] S. S. Margulies, L. E. Thibault, and T. A. Gennarelli, "Physical model simulations of brain injury in the primate," Journal of Biomechanics, vol. 23, no. 8, pp. 823-836, 1990.

[16] G. S. Nusholtz, P. S. Kaiker, and W. S. Gould, "Two factors critical in the pressure response of the impacted head," Aviation, Space, and Environmental Medicine, vol. 58, no. 12, pp. 1157-1164, 1987.

[17] L. Zhang, K. H. Yang, and A. I. King, "Biomechanics of neurotrauma," Neurological Research, vol. 23, no. 2-3, pp. 144-156, 2001.

[18] R. W. G. Anderson, C. J. Brown, P. C. Blumbergs, A. J. McLean, and N. R. Jones, "Impact mechanics and axonal injury in a sheep model," Journal of Neurotrauma, vol. 20, no. 10, pp. 961-974, 2003.

[19] J. Goeller, A. Wardlaw, D. Treichler, J. O'Bruba, and G. Weiss, "Investigation of cavitation as a possible damage mechanism in blast-induced traumatic brain injury," Journal of Neurotrauma, vol. 29, no. 10, pp. 1970-1981, 2012.

[20] J. E. Leestma, Forensic Neuropathology, CRC Press, Boca Raton, Fla, USA, 2nd edition, 2008.
[21] G. S. Nusholtz, E. B. Wylie, and L. G. Glascoe, "Internal cavitation in simple head impact model," Journal of Neurotrauma, vol. 12, no. 4, pp. 707-714, 1995.

[22] M. Oehmichen, R. N. Auer, and H. G. König, Forensic Neuropathology and Associated Neurology, Springer, Heidelberg, Germany, 2009.

[23] M. B. Panzer, B. S. Myers, B. P. Capehart, and C. R. Bass, "Development of a finite element model for blast brain injury and the effects of CSF cavitation," Annals of Biomedical Engineering, vol. 40, no. 7, pp. 1530-1544, 2012.

[24] S. A. Eucker, C. Smith, J. Ralston, S. H. Friess, and S. S. Margulies, "Physiological and histopathological responses following closed rotational head injury depend on direction of head motion," Experimental Neurology, vol. 227, no. 1, pp. 79-88, 2011.

[25] E. J. Pellman, D. C. Viano, A. M. Tucker et al., "Concussion in professional football: reconstruction of game impacts and injuries," Neurosurgery, vol. 53, no. 4, pp. 799-814, 2003.

[26] S. Rowson and S. M. Duma, "Brain injury prediction: assessing the combined probability of concussion using linear and rotational head acceleration," Annals of Biomedical Engineering, vol. 41, no. 5, pp. 873-882, 2013.

[27] A. A. Weaver, K. A. Danelson, and J. D. Stitzel, "Modeling brain injury response for rotational velocities of varying directions and magnitudes," Annals of Biomedical Engineering, vol. 40, no. 9, pp. 2005-2018, 2012.

[28] R. H. Pudenz and C. H. Shelden, "The lucite calvarium-a method for direct observation of the brain; cranial trauma and brain movement," Journal of Neurosurgery, vol. 3, no. 6, pp. 487505, 1946.

[29] Y. Feng, T. M. Abney, R. J. Okamoto, R. B. Pless, G. M. Genin, and P. V. Bayly, "Relative brain displacement and deformation during constrained mild frontal head impact," Journal of the Royal Society Interface, vol. 7, no. 53, pp. 1677-1688, 2010.

[30] E. B. Yan, V. P. A. Johnstone, D. S. Alwis, M.-C. MorgantiKossmann, and R. Rajan, "Characterising effects of impact velocity on brain and behaviour in a model of diffuse traumatic axonal injury," Neuroscience, vol. 248, pp. 17-29, 2013.

[31] V. R. Hodgson, E. S. Gurdjian, and L. M. Thomas, "Experimental skull deformation and brain displacement demonstrated by flash x-ray technique.," Journal of Neurosurgery, vol. 25, no. 5, pp. 549-552, 1966.

[32] H. Zou, J. P. Schmiedeler, and W. N. Hardy, "Separating brain motion into rigid body displacement and deformation under low-severity impacts," Journal of Biomechanics, vol. 40, no. 6 , pp. 1183-1191, 2007.

[33] R. K. Gupta and A. Przekwas, "Mathematical models of blastinduced TBI: current status, challenges, and prospects," Frontiers in Neurology, vol. 4, pp. 1-21, 2013.

[34] U. Krave, S. Höjer, and H.-A. Hansson, "Transient, powerful pressures are generated in the brain by a rotational acceleration impulse to the head," European Journal of Neuroscience, vol. 21, no. 10, pp. 2876-2882, 2005.

[35] U. Krave, M. Al-Olama, and H.-A. Hansson, "Rotational acceleration closed head flexion trauma generates more extensive diffuse brain injury than extension trauma," Journal of Neurotrauma, vol. 28, no. 1, pp. 57-70, 2011.

[36] E. Gutierrez, Y. Huang, K. Haglid et al., "A new model for diffuse brain injury by rotational acceleration: I. model, gross appearance, and astrocytosis," Journal of Neurotrauma, vol. 18, no. 3, pp. 247-257, 2001. 
[37] S. Rowson, S. M. Duma, J. G. Beckwith et al., "Rotational head kinematics in football impacts: An injury risk function for concussion," Annals of Biomedical Engineering, vol. 40, no. 1, pp. $1-13,2012$.

[38] J. W. Shek, G. Y. Wen, and H. M. Wisniewski, Atlas of the Rabbit Brain and Spinal Cord, Karger, Basel, Switzerland, 1986.

[39] P. Blumbergs, P. Reilly, and R. Vink, “Trauma," in Greenfields Neuropathology, S. Love, D. N. Louis, and D. W. Ellison, Eds., pp. 733-832, Arnold, London, UK, 8th edition, 2008.

[40] A. Hamberger, Y.-L. Huang, H. Zhu et al., "Redistribution of neurofilaments and accumulation of $\beta$-amyloid protein after brain injury by rotational acceleration of the head," Journal of Neurotrauma, vol. 20, no. 2, pp. 169-178, 2003.

[41] M. Runnerstam, F. Bao, Y.-L. Huang et al., "A new model for diffuse brain injury by rotational acceleration: II. Effects on extracellular glutamate, intracranial pressure, and neuronal apoptosis," Journal of Neurotrauma, vol. 18, no. 3, pp. 259-273, 2001.

[42] L. E. Goldstein, A. M. Fisher, C. A. Tagge et al., "Chronic traumatic encephalopathy in blast-exposed military veterans and a blast neurotrauma mouse model," Science Translational Medicine, vol. 4, no. 134, Article ID 134ra60, 2012.

[43] M. S. Chafi, G. Karami, and M. Ziejewski, "Biomechanical assessment of brain dynamic responses due to blast pressure waves," Annals of Biomedical Engineering, vol. 38, no. 2, pp. 490504, 2010.

[44] A. Säljö, F. Bao, K. G. Haglid, and H.-A. Hansson, "Blast exposure causes redistribution of phosphorylated neurofilament subunits in neurons of the adult rat brain," Journal of Neurotrauma, vol. 17, no. 8, pp. 719-726, 2000.

[45] A. Säljö, Y.-L. Huang, and H.-A. Hansson, "Impulse noise transiently increased the permeability of nerve and glial cell membranes, an effect accentuated by a recent brain injury," Journal of Neurotrauma, vol. 20, no. 8, pp. 787-794, 2003.

[46] R. C. Turner, Z. J. Naser, A. F. Logsdon et al., "Modeling clinically relevant blast parameters based on scaling principles produces functional \& histological deficits in rats," Experimental Neurology, vol. 248, pp. 520-529, 2013.

[47] C. Giordano, R. J. H. Cloots, J. A. W. van Dommelen, and S. Kleiven, "The influence of anisotropy on brain injury prediction," Journal of Biomechanics, vol. 47, no. 5, pp. 1052-1059, 2014.

[48] A. Suneson, H.-A. Hansson, and T. Seeman, "Pressure wave injuries to the nervous system caused by high-energy missile extremity impact: Part II. Distant effects on the central nervous system - a light and electron microscopic study of pigs," Journal of Trauma, vol. 30, no. 3, pp. 295-306, 1990.

[49] A. Suneson, H. A. Hansson, E. Lycke, and T. Seeman, "Pressure wave injuries to rat dorsal root ganglion cells in culture caused by high-energy missiles," Journal of Trauma, vol. 29, no. 1, pp. $10-18,1989$.

[50] J. J. Iliff, M. Wang, Y. Liao et al., "A paravascular pathway facilitates CSF flow through the brain parenchyma and the clearance of interstitial solutes, including amyloid $\beta$," Science Translational Medicine, vol. 4, no. 147, Article ID 147ra111, 2012.

[51] J. Nolte, The Human Brain, Mosby, St. Louis, Mo, USA, 6th edition, 2008.

[52] M. L. Rennels, T. F. Gregory, O. R. Blaumanis, K. Fujimoto, and P. A. Grady, "Evidence for a paravascular fluid circulation in the mammalian central nervous system, provided by the rapid distribution of tracer protein throughout the brain from the subarachnoid space," Brain Research, vol. 326, no. 1, pp. 47-63, 1985.
[53] V. R. Thrane, A. S. Thrane, B. A. Plog et al., "Paravascular microcirculation facilitates rapid lipid transport and astrocyte signaling in the brain," Scientific Reports, vol. 3, article 2582, 2013.

[54] R. O. Weller, E. Djuanda, H.-Y. Yow, and R. O. Carare, "Lymphatic drainage of the brain and the pathophysiology of neurological disease," Acta Neuropathologica, vol. 117, no. 1, pp. 1-14, 2009.

[55] J. Ho and S. Kleiven, "Can sulci protect the brain from traumatic injury?” Journal of Biomechanics, vol. 42, no. 13, pp. 2074-2080, 2009. 

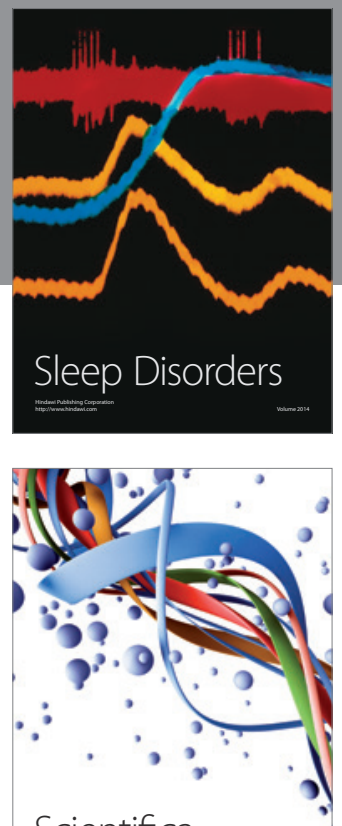

Scientifica
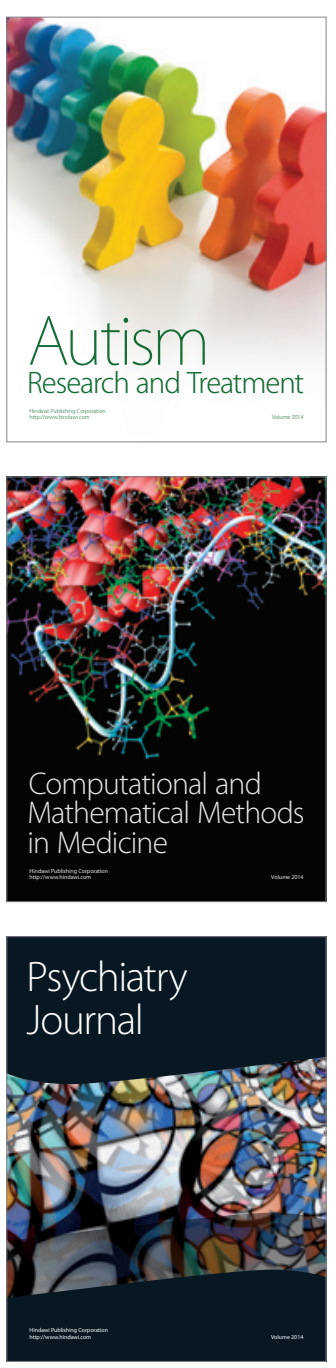
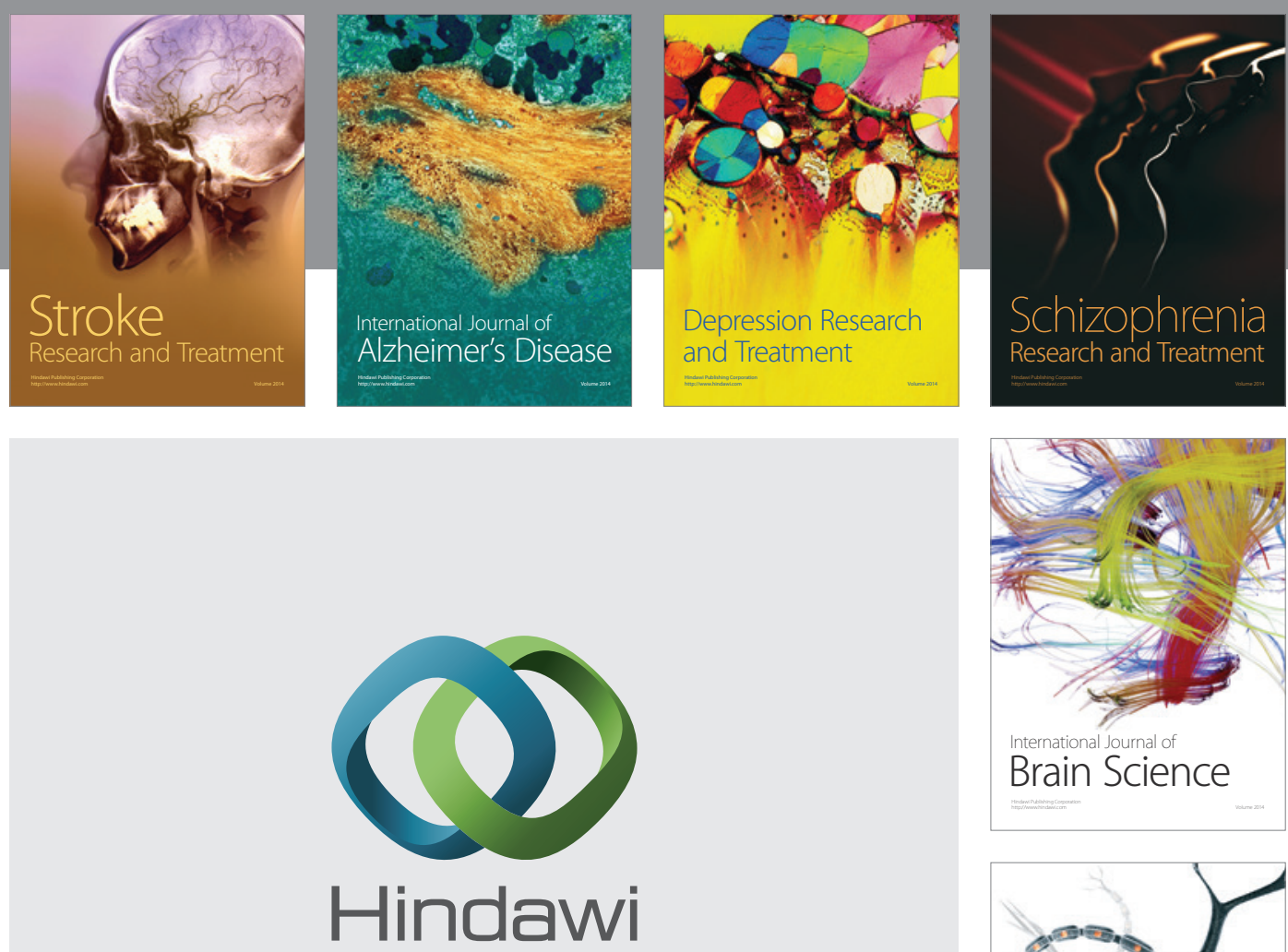

Submit your manuscripts at

http://www.hindawi.com
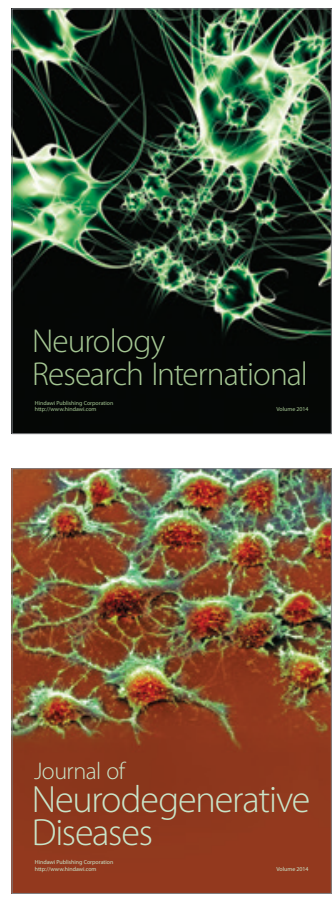

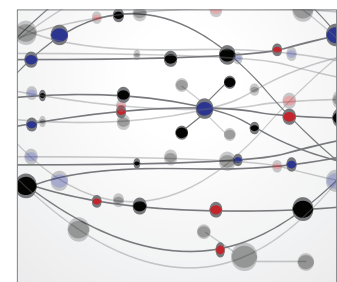

The Scientific World Journal
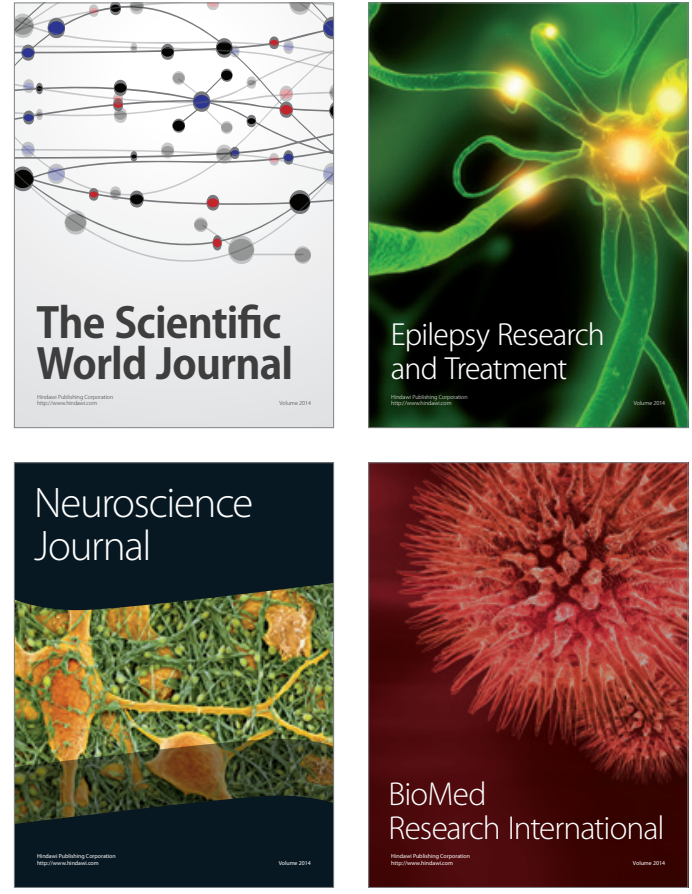

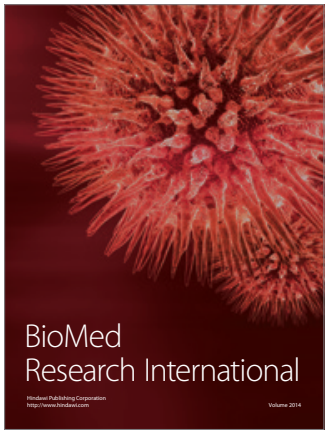

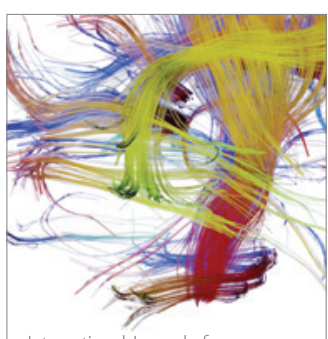

Brain Science

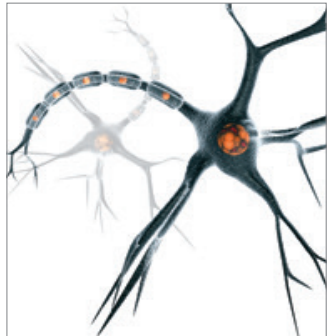

Neural Plasticity
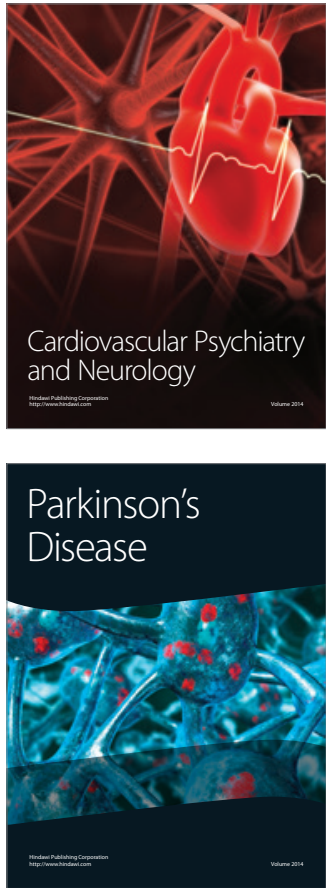